PO030

\title{
A STUDY ON COMPREHENSIBILITY OF INFORMATION OF INDUCTION SIGNS: DEGREE OF INFORMATION SEPARATION IN SIGNBOARDS
}

\author{
Marina Nishikawa
}

DOI 10.25039/x46.2019.PO030

from

CIE x046:2019

Proceedings

of the

29th CIE SESSION

Washington D.C., USA, June 14 - 22, 2019

(DOI 10.25039/x46.2019)

The paper has been presented at the 29th CIE Session, Washington D.C., USA, June 14-22, 2019. It has not been peer-reviewed by CIE.

\section{(C) CIE 2019}

All rights reserved. Unless otherwise specified, no part of this publication may be reproduced or utilized in any form or by any means, electronic or mechanical, including photocopying and microfilm, without permission in writing from CIE Central Bureau at the address below. Any mention of organizations or products does not imply endorsement by the CIE.

This paper is made available open access for individual use. However, in all other cases all rights are reserved unless explicit permission is sought from and given by the CIE.

CIE Central Bureau

Babenbergerstrasse 9

A-1010 Vienna

Austria

Tel.: +4317143187

e-mail: ciecb@cie.co.at

www.cie.co.at 


\title{
A STUDY ON COMPREHENSIBILITY OF INFORMATION OF INDUCTION SIGNS: DEGREE OF INFORMATION SEPARATION IN SIGNBOARDS
}

\author{
Nishikawa, M. ${ }^{1}$, Hirate, K. ${ }^{1}$ \\ 1 The University of Tokyo, Tokyo, JAPAN \\ 5091496603@mail.ecc.u-tokyo.ac.jp
}

DOI 10.25039/x46.2019.PO030

\begin{abstract}
A lot of induction signs are set up in places where people gather. These provide users with information to get to their destination quickly and safely. In order to convey these contents correctly, it is important to make the information easy to understand. If there are specific rules on the board, it will be helpful to make it easy to understand even if the contents are complicated. In this study, we focused on the separation of information by the margin in the sign. An experiment was carried out to adjust the width between two destination information in the signboard. It was possible to show unitarily the threshold of the width for recognizing "two adjacent pieces of information are separate pieces of information" and how different the separations were from each other, such as borders and colour coding.
\end{abstract}

Keywords: Sign, Comprehension on information, Colour

\section{Introduction}

A lot of induction signs are set up in places where people gather, such as streets, public transportation facilities, and commercial facilities. These provide users with information to get to their destination quickly and safely. In order to correctly convey the contents of these signs, it is important to make the information on the board quick and easy to understand. For public transportation, Guideline (Foundation for Promoting Personal Mobility and Ecological Transportation, 2002) is shared and each company has its own regulations on dimensions such as character size and margin width. In the field of design, there is a common rule for proper alignment and margin (Williams, 2014). However, these are mainly aimed at readability and aesthetic beauty. In many cases, there are no specific indicators, and there are few indicators that can be referenced in places with few regulations such as commercial facilities. There are few bad things if it is a space with a definite rule or signs with a small amount of information, but there is also a sign that carries a lot of information and these are mixed. If there is a specific rule in the board, it is thought that it will help to make the signboards easy to understand even complex contents.

In this research, we focus on separation of information by margin and aim to confirm how the degree of information separation changes by division of area in one board.

\section{Methods}

In this experiment, the subject had to be able to easily adjust the position of the information. The editing screen of Adobe Illustrator was used. The edit screen is displayed on the notebook PC, the adjustment part was selected, and the left and right positions would be adjusted with the arrow keys. The position of the adjustment part recorded the position information displayed on the editing screen. The position information was hidden from the subjects. The images used in the experiment were referred to the induction sign posted at the station in Japan. It was written in four languages: Japanese, English, Chinese, Korean. Fonts in each language, size ratio and line spacing were the same as existing ones. (Figure 1) The subjects were 20 Japanese people in their 20s (8 males and 12 females). 


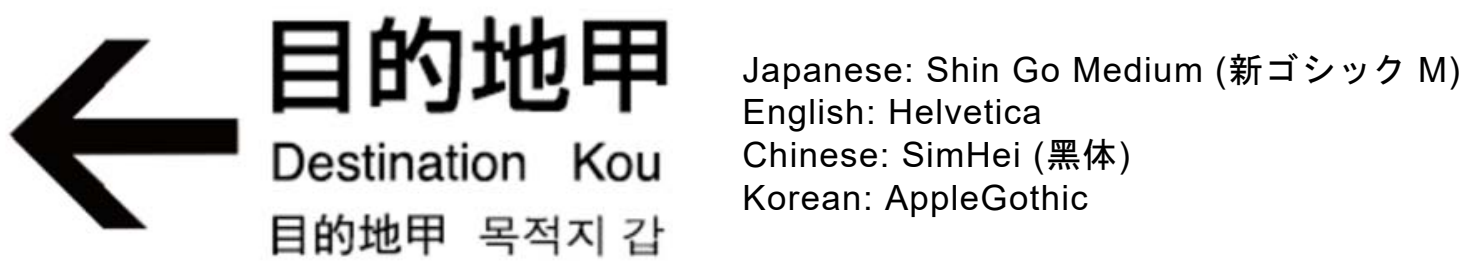

Figure 1 - Information used in the experiments and fonts of each language

\subsection{Experiment 1}

Two pieces of destination information (destination Kou and destination Otsu) on a white background were displayed. The position of the adjustment part (the information on the right side) was adjusted so that it would be "just understood" and "clearly understood" that they were other information. These were adjusted in ascending (widening) and descending (narrowing) series. The destination information on the right side had two types of arrow direction: "up" and "right". (Figure 2)

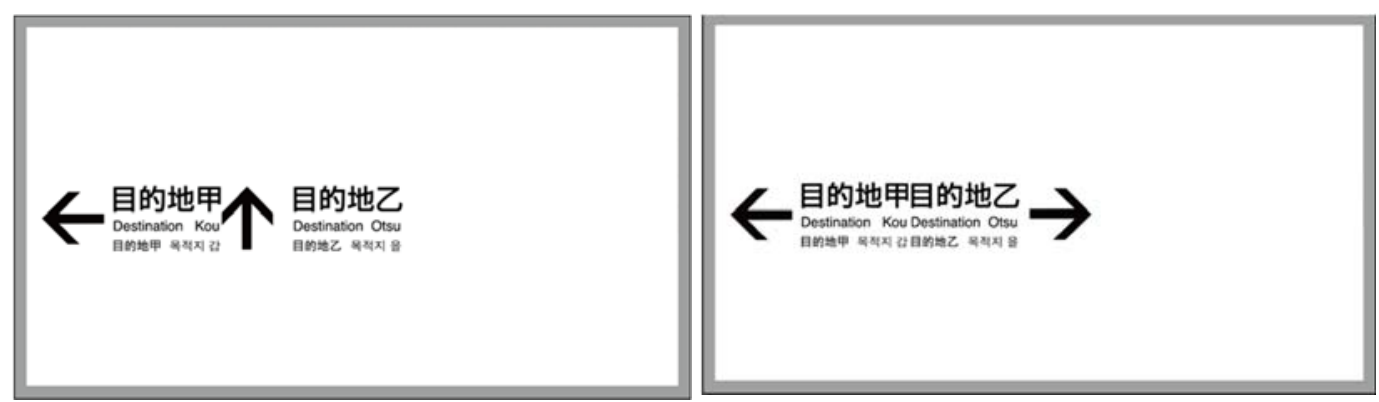

Figure 2 - Screen of Experiment 1 ("up" and "right")

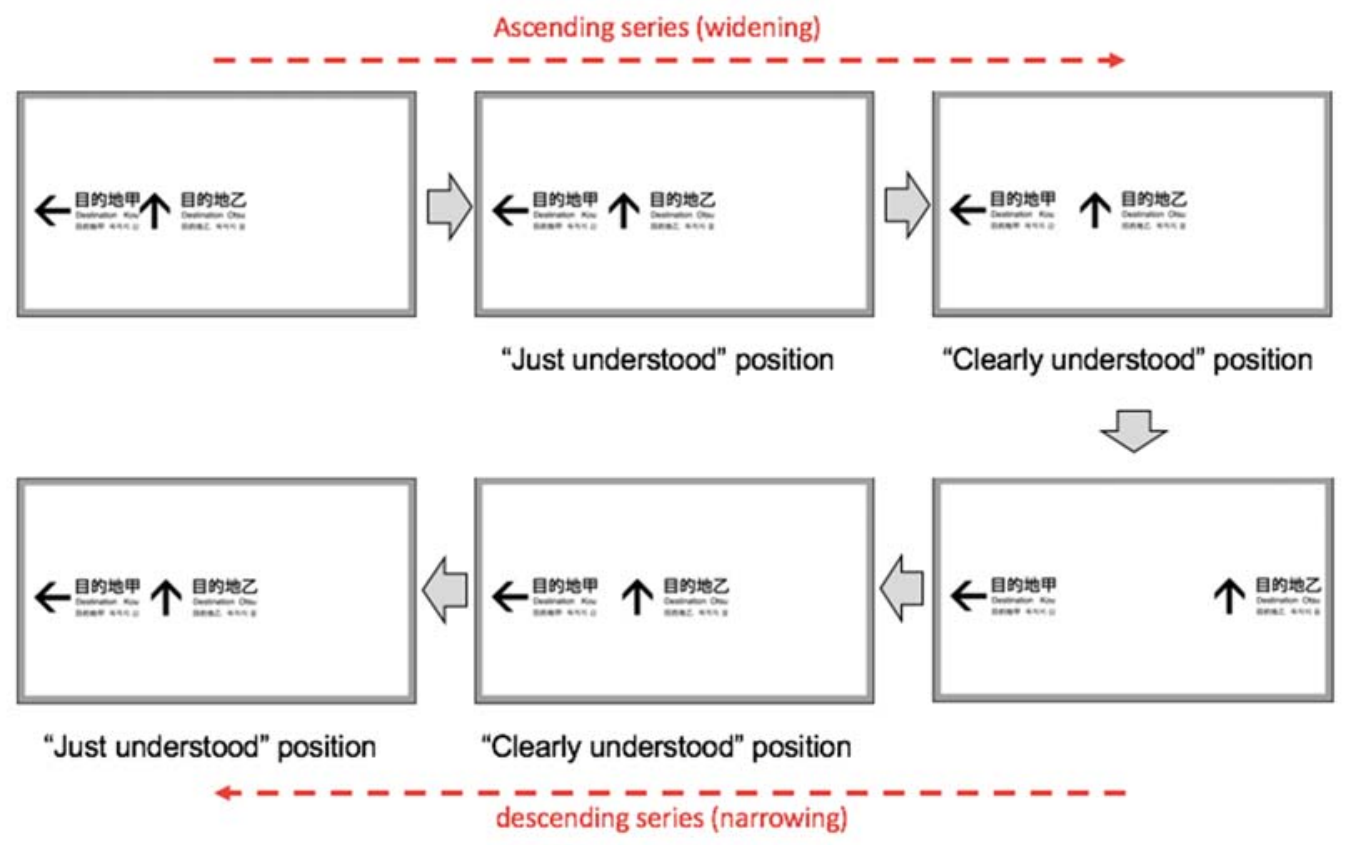

Figure 3 - Flow of Experiment 1 


\subsection{Experiment 2}

In the upper half of the screen, two pieces of destination information (destination instep and destination point) using various divisions were displayed. The background was a photograph of the ceiling to imitate the sign hung on the ceiling of the station. The same destination information as the upper half was displayed on the white background in the lower half of the screen. The position of the adjustment part (the information on the right) was adjusted so that the degree of separation of the information became equal at the upper half and lower half. (Figure 4) These were adjusted in ascending (widening) and descending (narrowing) series. There were four types of information in the upper half image. (Figure 5) The two pieces of information were on separated plate, the border lines (thick, medium, thin), and colour coding (white, yellow, green, black). The thickness of the border was the same as that of the sign frame, the medium was $1 / 2$, and the thin was $1 / 4$. For the medium and the thin, it was prepared shorter border line did not touch the frame. In addition to white and black, yellow used for the exit, and green was as the colour of brightness between black and yellow were prepared. Table 1 shows the luminance of each colour and ratios of each colour combination.
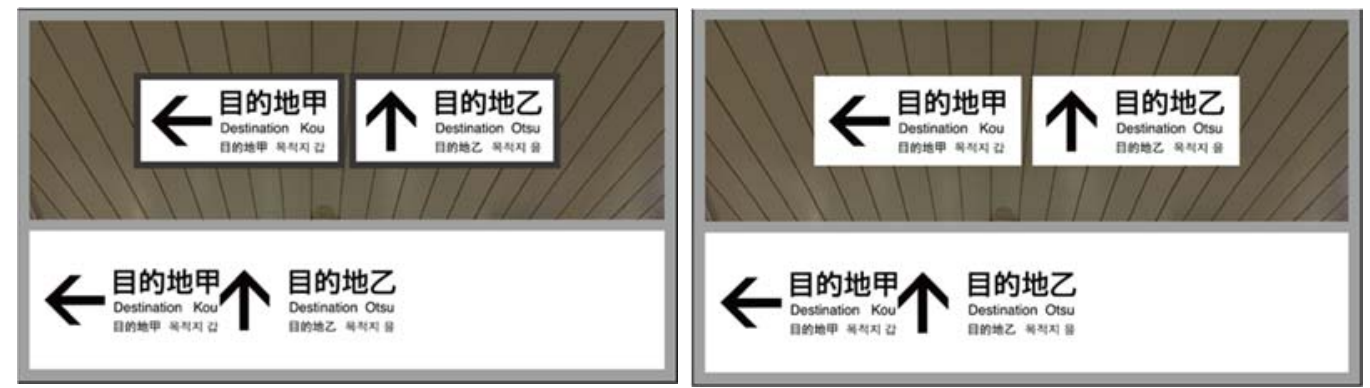

Figure 4 - Screen of Experiment 2 (separated plate: with frame and without frame)
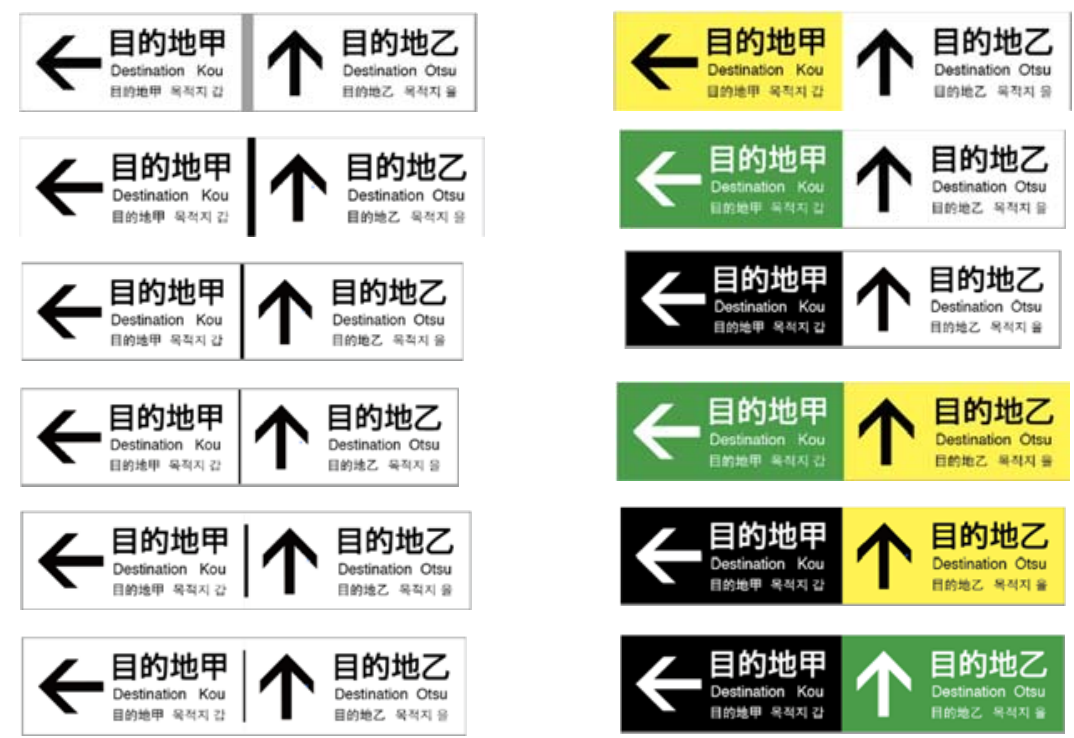

Figure 5 - How to divide information in Experiment 2

\section{Results}

\subsection{Experiment 1}

The average ratio of adjustment width to height of information in each image is shown in Figure 7. Analysis of variance for the position and orientation of the arrows showed no statistically significant difference. Analysis of variance was performed on the adjustment range of "just understood" and "clearly understood", and statistically significant differences were found. 
Table 1 - The luminance ratio of each colour combination in Experiment 2

\begin{tabular}{|l|l|l|l|}
\hline & $\begin{array}{l}\text { Luminance of } \\
\text { bright colour } \\
\left(\mathrm{cd} / \mathrm{m}^{2}\right)\end{array}$ & $\begin{array}{l}\text { Luminance of } \\
\text { dark colour } \\
\left(\mathrm{cd} / \mathrm{m}^{2}\right)\end{array}$ & Luminance ratio \\
\hline White and Yellow & 323 & 292 & 1.11 \\
\hline Yellow and Green & 292 & 131 & 2.22 \\
\hline White and Green & 323 & 131 & 2.46 \\
\hline Green and Black & 131 & 19.9 & 6.60 \\
\hline Yellow and Black & 292 & 19.9 & 14.7 \\
\hline White and Black & 323 & 19.9 & 16.2 \\
\hline
\end{tabular}

Ascending series (widening)

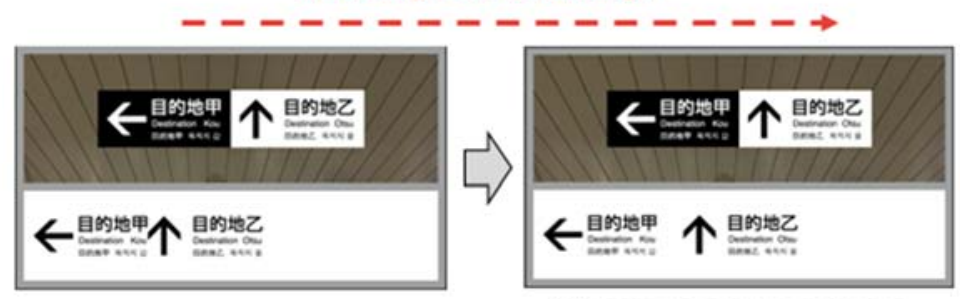

"The degree of separation is equal" position
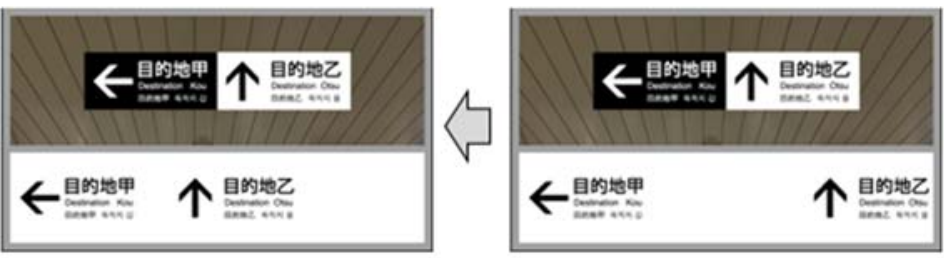

"The degree of separation is equal" position

$$
\leftarrow---------------
$$

Figure 6 - Flow of Experiment 2

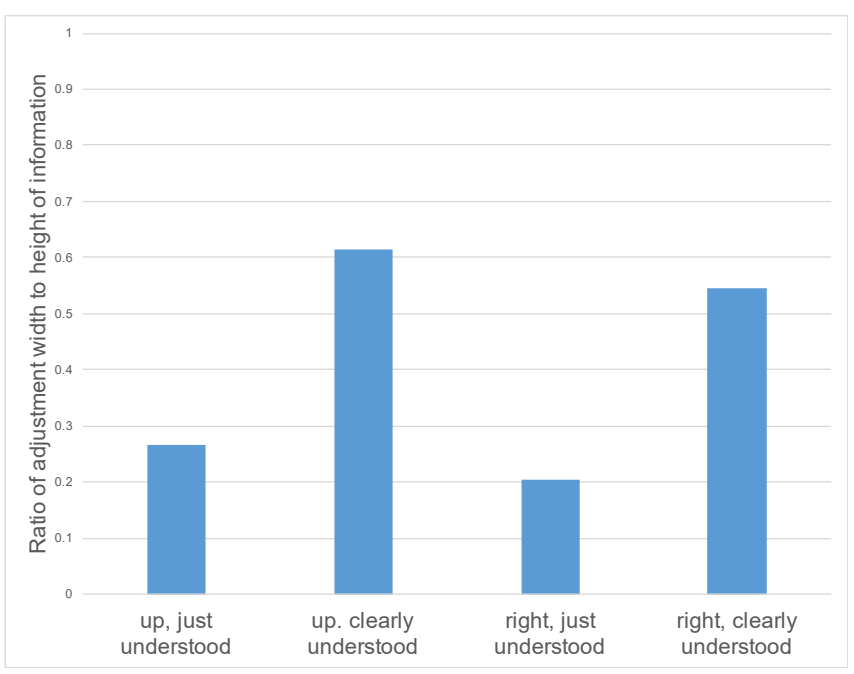

Figure 7 - The average ratio of adjustment width to height of information 


\subsection{Experiment 2}

Figure 8 shows the result of the adjusted width ratio to the original width in each image. When a test was performed against the distribution assuming an average of 1 , significant differences were found in all images. Since there was no significant difference between the left and right in the colour coding, they were averaged.

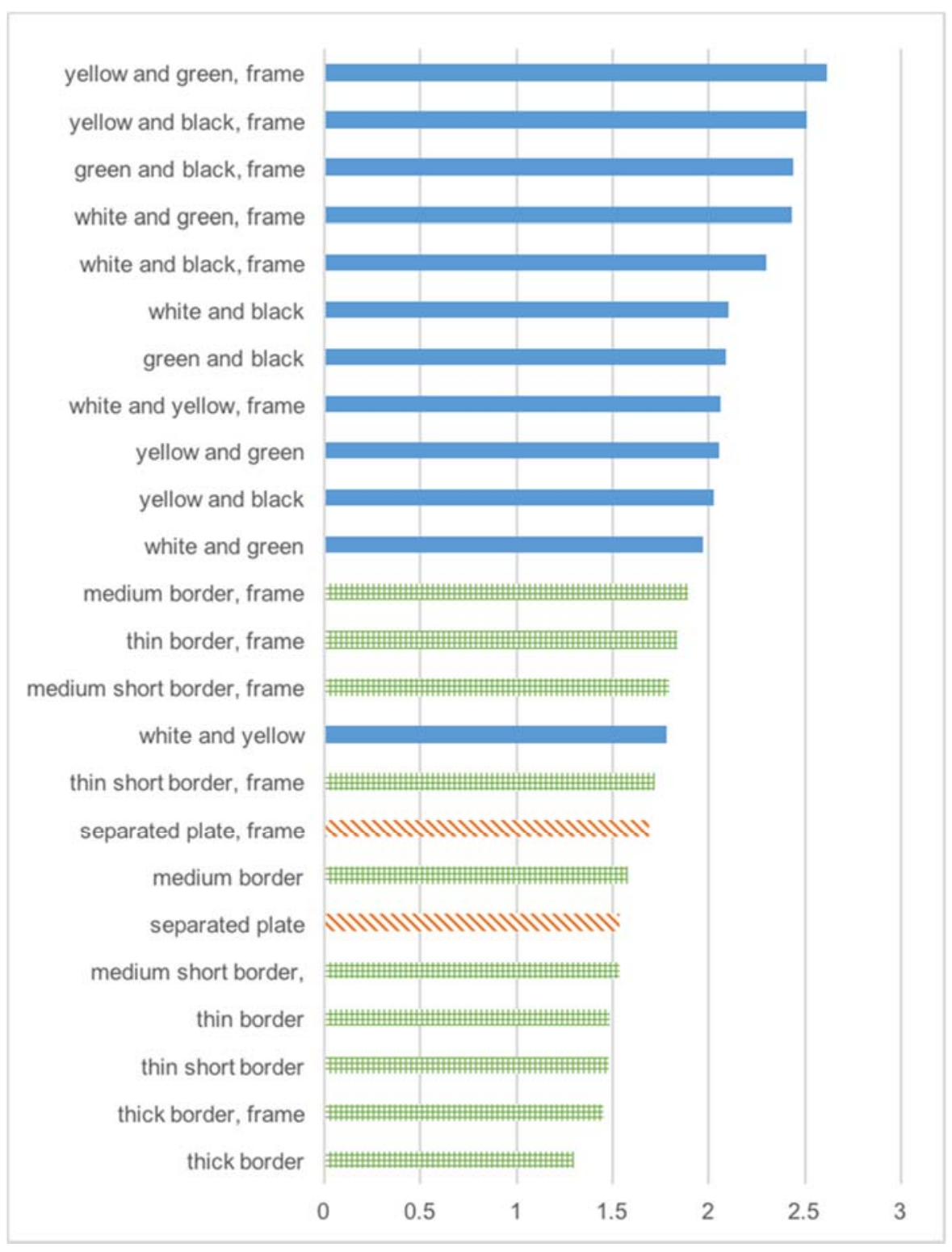

Figure 8 - Result of Experiment 2 (ratio of adjustment width to original widthfor each division)

\section{Discussions}

From the results of Experiment 1, it was found that in this design, it was recognized as a mass of information regardless of the position and direction of the arrow. The ratios of the adjustment range to the height of the destination information are 0.26 for "just understood" and 0.62 for "clearly understood". These can be said to be the width threshold for recognizing that "two adjacent pieces of information are separate pieces of information". The adjustment range for "clearly understandable" is twice that of "very clearly understood". If the area of the signboard is large, it is considered that the wider the information width, the easier for users to understand the information.

In Experiment 2, the width of the separated plates was adjusted to about 1.6 times, that using the borderline was about 1.3 to 1.9 times, and that using the colour was about 1.8 to 2.6 times. 
It can be said that this value represents the strength of each degree of separation. The reason for the lower result of the separated plate is considered to be that the background of this experiment was that there was no three-dimensional effect and it was not felt that the plate was separated. The width was adjusted significantly wider in the frame compared to the frameless. This tendency is particularly pronounced in colour coding. This is considered to be due to the fact that the different information is used in the frame and the other information is emphasized. The thick border was adjusted to be wider without a frame than with a frame. This is considered that the border was recognized as a part of the frame and did not function as the border. As compared with colour coding, only the width of the combination of white and yellow was adjusted to be narrow. The other combinations did not make much difference. The luminance ratios were 1.1 for white and yellow, 2.2 for yellow and green, and 2.4 to 16 for the other combinations. (Table 1) If the luminance ratio is twice or more, it seems to be judged that it is "another information".

\section{Conclusion}

In this research, it was showed unitarily how the degree of separation differed depending on how the areas were divided by adjusting the width of the two pieces of information. Colour coding has a strong power, but its effect does not change if its luminance ratio is over a certain level. It is not always better for the border to be thicker, and it is necessary to consider the relationship with the entire signature such as the frame. This time, the image which displayed two information side by side was used, and it does not consider about the signboard of different layout. Also, it was not considered a design in which colour coding and boundaries coexist. In addition, there were no subjects with different colour vision, and it was not considered the difference due to the colour vision characteristics of the degree of separation for colour. In future research, we would like to examine signatures containing three or more pieces of information and to verify the appropriateness of separation using images of actual signs.

\section{Acknowledgements}

This research was supported by the research grant of Toda Scholarship Foundation.

\section{References}

Foundation for Promoting Personal Mobility and Ecological Transportation (Supervised by the Ministry of Land, Infrastructure, Transport and Tourism), 2002. Sign system guidebook for public transport and passenger organizations, Tokyo: Taisei Publishing Co. (written in Japanese)

WILLIAMS, R. 2014. The Non-Designer's Design Book, 4th Ed. San Francisco: Peachpit Press,

IKEDA Y. 2018. A study on sign plan at railway stations based on user evaluation, PhD Thesis, University of Tokyo, Tokyo (written in Japanese) 